\title{
Exploring New Strategies to Monitor Autophagy and Related Cell Death Path- ways Using Raman Spectroscopy
}

\author{
Martin Isabelle ${ }^{1,2}$, Vincent I Poon ${ }^{2}$, Zoe V Petropoulos ${ }^{3}$, Samantha J Harder ${ }^{1}$, Julian J Lum ${ }^{2,3^{*}}$ \\ ${ }^{1}$ Department of Physics and Astronomy, University of Victoria, 3800 Finnerty Rd, Victoria, BC V8P 5C2, Canada \\ ${ }^{2}$ Trev and Joyce Deeley Research Centre, British Columbia Cancer Agency, 2410 Lee Avenue, Victoria, BC, V8R \\ 6V5, Canada \\ ${ }^{3}$ Department of Biochemistry and Microbiology, University of Victoria, 3800 Finnerty Rd, Victoria, BC V8P 5C2, \\ Canada
}

${ }^{\star}$ Corresponding author: Julian J Lum, Trevand Joyce Deeley Research Centre, British Columbia Cancer Agency, 2410 Lee Avenue, Victoria, BC, V8R 6V5, Canada; E-mail: JLum@bccancer.bc.ca

Received Date: July 20, 2013 Accepted Date: August 20, 2013 Published Date: August 22, 2013

Citation: Martin Isabelle, et al. (2013) Exploring New Strategies to Monitor Autophagy and Related Cell Death Pathways Using Raman Spectroscopy. J Cancer Res Therap Oncol 1: 1-5

\section{Autophagy in the context of cancer}

Macroautophagy, (heretofore referred to as autophagy) is thought to play a pivotal role in tumorigenesis and tumor cell survival. Autophagy is a self-degradative cellular process that recycles intracellular components such as proteins and lipids, and may also be used to clear extraneous or damaged organelles. Through this process, metabolites such as amino acids or fatty acids can be liberated for use by the cell. While basal levels of autophagy are required for cellular homeostasis, autophagy is most commonly induced in response to metabolic stressors. In this way, autophagy may be utilized by cancer cells to adapt to the tumor microenvironment, which may be hypoxic and/or nutrient low, or as an adaptive response to cytotoxic insult by chemotherapeutic agents. Conversely, excessive or dysregulated autophagy may be part of the cell death pathway continuum, having been previously implicated in both apoptosis and necrosis [1,2]. In addition, down regulation of tumor autophagy may promote immune evasion by disrupting the antigen-processing pathway $[3,4]$. Therefore, further investigation of autophagy's role in tumor cell survival and death is warranted.

\section{Mechanism of action in autophagy}

At each step of autophagy, a highly ordered and defined biochemical reorganization is executed, and these changes may be used as markers to detect autophagy. The mammalian Target of Rapamycin (mTOR) has a central role in the nutrientsensing pathway, and serves to inhibit autophagy via phosphorylation of the autophagy-initiating ULK1/2 complex in times of plenty [5]. Stressors such as hypoxia, nutrient depletion or DNA damage lead to inhibition of mTOR activity,

C2013 The Authors. Published by the JScholar under the terms of the Creative Commons Attribution License http://creativecommons.org/licenses/ by/3.0/, which permits unrestricted use, provided the original author and source are credited. as well as activity of the autophagy-activating Beclin1 protein, resulting in a large shift in cellular programming [6]. However, autophagy regulation is complex, and involves a number of cellular pathways including the modulation of transcription factors and post-translational protein modification [7]. Regardless, upon initiation of autophagy, the microtubule-associated protein 1A/1B-light chain 3 (LC3) undergoes a cleavage event, denoted as a shift from LC3-I to LC3-II [8]. LC3-II then associates with phosphatidylethanolamine bound to both sides of the membrane, which promotes asymmetric membrane curvature, and after further elongation of the membrane, helps form the autophagic vesicle (the autophagosome). During formation, the autophagosome may non-selectively engulf cytosolic components, or selectively traffic contents to be degraded, such as lipid droplets, damaged organelles or misfolded proteins [9-12]. Finally, the autophagosome eventually fuses with a lysosome in a $\mathrm{pH}$-dependent manner, after which acid hydrolases breakdown the vesicle components, releasing metabolites back into the cytosol.

\section{Current methods and challenges in au- tophagy detection}

Autophagy has been assessed using electron microscopy, fluorescence microscopy and immunoblotting of autophagy related proteins [13-17]. In vitro, the most common approach to measure autophagy induction is using the autophagic flux assay, where changes in the cleavage patterns of LC3 are monitored. Generally, LC3-II is measured via immunofluorescent microscopy or Western blotting; in cells treated with an autophagic stimulus, degradation of autophagosomal contents will result in a decrease of LC3-II. In cells treated with lysosomotropic fusion or degradation inhibitors such as chloroquine, LC3-II will accumulate relative to LC3-I, and this process can be visualized as an increase in punctate staining of LC3-II, signifying autophagic vesicles, relative to normal cytosolic distribution of LC3. Less commonly used assays make use of tech- 
niques to measure the turnover of cytosolic proteins, or the activity of transcriptional regulators, such as mTOR. Ex vivo, the current gold standard for characterizing autophagy is the use of transmission electron microscopy to identify autophagic vesicles. Formalin-fixed paraffin-embedded tissue sections may also be used to, probe for changes in autophagy-related proteins such as Beclin1 or LC3a [18]. However, the resolution of immunohistochemical staining is currently limited, and due to other biological roles of Beclin and LC3, the data collected thus far using these assays is correlative.

All of these techniques are invasive and require some modification of the cells either using a fluorescent reporter construct (e.g GFP-LC3 constructs), fixation, staining or samples being placed in a vacuum. They are also expensive, time-consuming, and require operation of equipment by highly skilled personnel. There is a significant need, especially for primary human tissues, for a highly specific and sensitive marker for autophagy. In this commentary, we provide a rationale to explore using Raman Spectroscopy (RS) for this purpose.

\section{Applications of Raman Spectroscopy in cellular systems}

Spectroscopy, the study of the interaction of electromagnetic radiation with matter, is an important biochemical tool that can be used to probe the structure and composition of molecules in a biological sample. There are many different types of spectroscopy used in biochemistry which include: emission spectroscopy (i.e. fluorescence and phosphorescence), absorption spectroscopy (e.g. infrared and ultraviolet-visible), elastic scattering and reflection spectroscopy, resonance spectroscopy (e.g. nuclear magnetic resonance), and inelastic scattering spectroscopy (i.e. Raman and Compton scattering). The most common types of spectroscopy used in biochemistry are fluorescence, infrared, visible, Ultraviolet (UV), and nuclear magnetic resonance spectroscopy. RS is a vibrational spectroscopic method in which monochromatic light incident on a sample is inelastically scattered, collected and dispersed via a grating spectrometer onto a detector. The energy required to excite a molecular vibration depends on the masses of the atoms involved in the vibration and the type of chemical bonds between these atoms. It also may be influenced by the structure, interactions and the chemical microenvironment of the molecule.

These vibrational signatures provide a "molecular fingerprint" of components within the biological sample related to proteins (e.g amino acids, conformational structures), nucleic acids (DNA and RNA), carbohydrates (e.g. glucose and glycogen) and lipids (e.g. cholesterol, choline, $\mathrm{CH} 2$ groups). It is the positions, relative intensities and shapes of these bands in a Raman spectrum that carry detailed information about the molecular composition of the sample. Advanced chemometric statistical analytical tools can be used to extract relevant biochemical information from large data sets or identify complex spectral trends.

RS has proven useful in a range of biological and medical applications. Particularly, RS has been utilized in oncology by offering an objective technique for diagnosis and monitoring of cellular processes. It has been used in the study and diagnostics of several types of cancer including skin [19], bladder [20], breast [21,22], and cervical [23]. Analysis of tissue samples, single cells and biological fluids is possible due to its versatile nature, and RS has been used for biochemical analysis of various cell processes including cell proliferation [24], differentiation [25], autophagy [26] and cell death [17]. Currently the most conventional form of RS used in medical research is dispersive and confocal Raman microspectroscopy for ex vivo applications. However, in the last decade, there have been a number of studies using Raman spectroscopy probes that do not require the removal of tissue or cells for analysis but instead, can be used as an in vivo diagnostic tool [27-29]. As well as those mentioned above, RS has additional advantages over current biomedical technologies that analyze biological cells and tissue. RS can be used in aqueous solutions, measurements need only a relatively short amount of time to be performed (10 to 30 seconds) and RS has higher resolving powers compared to its spectroscopic counterparts (e.g. $1 \mu \mathrm{m}$ spatial resolution in the most optimal system) which allows for intracellular and even subnuclear structural imaging and analysis of cells [30].

The main difficulties of Raman spectroscopy have come from its weak scattering signal compared to its counterpart, Rayleigh elastic scattering, which is $10^{7}$ times stronger in signal. In order to resolve this issue, modern Raman spectroscopic systems have incorporated technology such as multiple monochromators and Rayleigh holographic notch filters in order to reducing the elastic Rayleigh scattering, and improve signal to noise.

\section{Raman Spectroscopy in autophagy}

Konorov et al. [26] assessed RS in autophagy using metastatic prostate carcinoma cells and human breast carcinoma cells exposed to starvation conditions (glutamine deprivation and amino acid deprivation). Prior to RS cell measurements, fluorescence microscopy was used to assess the response of the cells to starvation conditions using the GFP-LC3 reporter construct. Increased numbers of GFP-LC3-positive puncta were clearly visible after one day of glutamine deprivation indicative of autophagosome formation during autophagy. In the MCF7 human breast carcinoma cells, the average autophagosome content per cell increased 2.9 times (from 9.1 to 26.4) in the absence of glutamine while metastatic prostate carcinoma cells increased 2.1 times (from 5.5 to 11.6). RS of the cells during starvation conditions revealed increases in the phospholipid $718 \mathrm{~cm}^{-1}$ Raman band, indicating accumulation of doublemembrane autophagic vesicles. There was also a decrease in the $937 \mathrm{~cm}^{-1}$ and $1003 \mathrm{~cm}^{-1}$ Raman bands potentially attributed to degradation of proteins due to autophagy, which is indicative of an autophagic response to starvation stimuli. The authors concluded that the intensity of the $718 \mathrm{~cm}^{-1}$ Raman band relative to the $784 \mathrm{~cm}^{-1}$ (DNA-associated, accounting for variation in cell cycle phase in cells) intensity could be a useful spectral marker for monitoring cellular response to starvation stimuli. As illustrated in Figure 1, various examples of biomolecular signatures may be assessed by Raman spectroscopy to indicate the different stages of autophagy. For example, formation of double membrane vesicles results in increases in phospholipid 


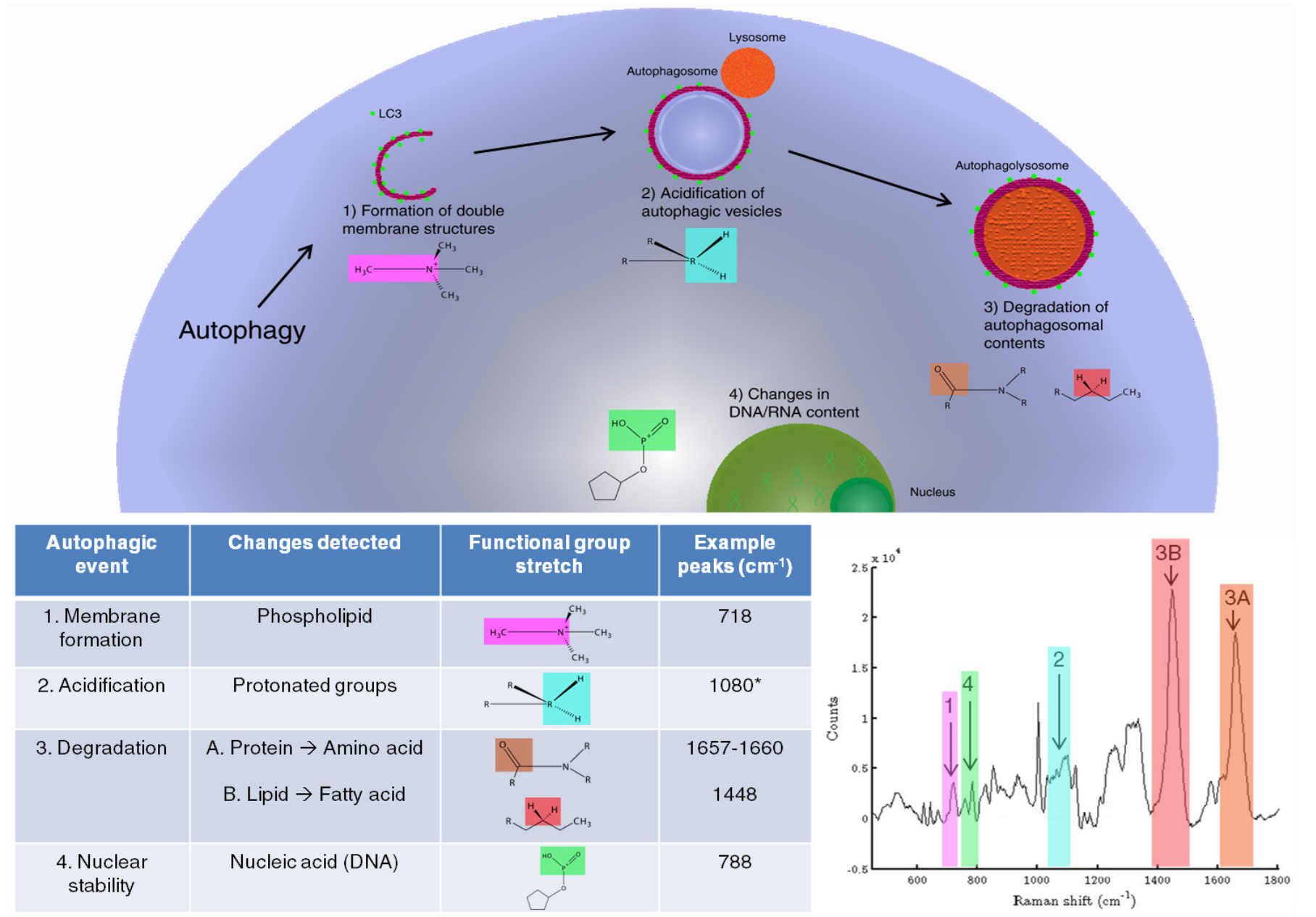

Figure 1 - Characteristics of Autophagy measurable via Raman Spectoscopy bands at $718 \mathrm{~cm}^{-1}$, while degradation of complex biomolecules (e.g proteins and lipids) into their constituent monomer units (amino acids, fatty acids and glycerol) are visualized by changes in peak intensities $\left(1657-1660 \mathrm{~cm}^{-1}\right.$ and $\left.1448 \mathrm{~cm}^{-1}\right)$.

\section{Raman Spectroscopy in apoptosis and ne- crosis}

Raman spectroscopy has also been used to study and discriminate apoptosis and necrosis in cancer cells. Apoptosis is the process of programmed cell death that involves a series of biochemical events leading to morphological changes including blebbing, loss of membrane attachment, cell shrinkage, nuclear fragmentation, chromatin condensation, chromosomal DNA fragmentation, and formation of apoptotic bodies. Apoptosis is usually beneficial to the organism, taking place during embryonic development or preventing tumor formation by acting as a homeostatic regulator of cell death and mitotic rates. Necrosis, in contrast, is the premature death of cells and tissue caused by factors external to cells (e.g. infection, toxin or trauma). Events such as organelle membrane disruption and hypoxia are triggers of necrosis, resulting in ATP depletion, metabolic collapse, cell swelling and rupture, leading to inflammation. Discrimination between apoptotic and necrotic cell death is technically challenging in vitro, and relies heavily upon pathologist experience in ex vivo settings.
In vitro, apoptosis may be detected through Annexin V staining, which binds to phosphotidylserine exposed during early stages of apoptosis, caspase activation, or DNA fragmentation assays; unfortunately, many of these markers are also seen in necrotic cells. From tissue sections, caspase cleavage and cellular morphology are commonly used to distinguish apoptosis and necrosis, and these remain qualitative measures. Thus, new techniques to distinguish these modes of cell death are needed.

Kunapareddy et al. [16] used RS to characterize necrotic cell death. Necrosis in human malignant melanoma cell line (MEL-28) was induced through a combination of oxygen and glucose deprivation. RS of nonproliferating live cells and dead cells were taken and basis spectra of protein, lipid, RNA, DNA, and glycogen were fitted to the each of the cell spectra. Decreases in the relative amounts of lipid $(717,935,1085,1448$, 2854 and $\left.2896 \mathrm{~cm}^{-1}\right)$ and RNA $\left(900,1092\right.$ and $\left.1575 \mathrm{~cm}^{-1}\right)$, and an increase in the relative protein content $(642,827,1395$ to $1425 \mathrm{~cm}^{-1}$ ) were observed in dead cells. As the authors indicated, this is consistent with the process of coagulative necrosis which features destruction of cytoplasmic structures and degradation of the membranes in the first stages of necrosis. There were also Raman band changes in protein amide I bands (from $1657 \mathrm{~cm}^{-1}$ in live cells to $1660 \mathrm{~cm}^{-1}$ in dead cells) indicating conformational and composition changes in protein and nucleic acid in dead cells. 
Apoptotic cell death was studied in RS by Verrier et al. [17] who used Raman microspectroscopy to assess molecular changes in human lung carcinoma epithelial cells (A549) induced into apoptotic cell death using Triton X-100. They observed decreases in DNA $\left(788 \mathrm{~cm}^{-1}\right)$ by $80 \%$ after 72 hours of treatment indicating phosphodiester bonds breakage as a result of fragmentation and disintegration of the DNA strands which was confirmed by DNA integrity assays. The authors also observed a 45\% decrease in protein $(632,645,854,938,1005$ and 1342 $\mathrm{cm}^{-1}$ ) explained by protein cleavage following caspase activation. An increase in lipid concentration was also found (1660 and $1743 \mathrm{~cm}^{-1}$ ). These lipid changes were also observed in Etoposide-treated (apoptotic) human breast cancer cells from another study by Zoladek [15] et al. Etoposide-treated cancer cells showed a high accumulation of both membrane and nonmembrane lipids (triglycerides) correlating to formation of large numbers of lipid vesicles in apoptotic cells. The increase in triglyceride non-membrane bound lipids in apoptotic cells was thought to be due to the inhibition of phosphatidylcholine biosynthesis, activation of phospholipases, and the formation of lipid bodies already seen in cells exposed to acidic $\mathrm{pH}$ $[31,32]$, cells engaged in inflammation [33] or apoptotic cells [34]. However contrary to findings by Verrier et al.[17] Raman bands assigned to DNA also increased in apoptotic cells, which was attributed to nuclear condensation.

$\mathrm{RS}$, in combination with machine learning chemometrics, has also been used to discriminate between apoptosis and necrotic cell death [35]. A549 lung epithelia cells were forced into necrosis (using Triton X-100) or apoptosis (using etoposide). Raman spectra were collected from both cell death states, and support vector machines (SVMs) machine learning was applied to test the ability of RS to classify correct states of cell death. SVM classifiers were successfully able to discriminate the control cells from cells treated with etoposide or Triton $\mathrm{X}-100$.

\section{Summary}

These Raman studies demonstrate the uniqueness of Raman spectroscopy to be able to obtain vast biochemical information (particularly lipid, protein and DNA relative intensity changes) from live and dead cancer cells non-invasively (as illustrated in Figure 1), without the use of fluorescent reporter constructs, labels, stains or contrasting-enhancing techniques. Differentiation of autophagy, apoptosis and necrotic cell death is important in biomedical sciences, especially in cancer treatment. Autophagy may suppress the initiation of cancer growth, promote cancer cell survival, or play a role in cell death. Therefore improved detection, discrimination and understanding of autophagy and related cell death processes will enable improved and optimized treatment plans for cancer therapy through assessing cellular response to chemotherapy, radiotherapy and other cancer-targeted treatments and differentiating between autophagy, apoptosis and necrosis occurring in cells.

\section{Acknowledgements}

Julian J Lum is supported by a New Investigator Award, Operating Grant and Team Grant (all from the CIHR).

\section{References}

1) Nikoletopoulou V, Markaki M, Palikaras K, Tavernarakis N (2013) Crosstalk between apoptosis, necrosis and autophagy. Biochim Biophys Acta.

2) Chaabane W, User SD, El-Gazzah M, Jaksik R, Sajjadi E, et al. (2013) Autophagy, apoptosis, mitoptosis and necrosis: interdependence between those pathways and effects on cancer. Arch Immunol Ther Exp 61: 43-58.

3) Yan Y, Liu N, Lu L, Zang CM, Shao B, et al. (2013) Autophagy enhances antitumor immune responses induced by irradiated hepatocellular carcinoma cells engineered to express hepatitis B virus X protein. Oncol Rep 30: 993-999.

4) Yi Y, Zhou Z, Shu S, Fang Y, Twitty C, et al. (2012) Autophagy-assisted antigen cross-presentation: Autophagosome as the argo of shared tumor-specific antigens and DAMPs. Oncoimmunology 1: 976-978.

5) Mizushima N (2010) The role of the Atg1/ULK1 complex in autophagy regulation. Curr Opin Cell Biol 22: 132-139.

6) Yang Z, Klionsky DJ (2010) Eaten alive: a history of macroautophagy. Nat Cell Biol 12: 814-822.

7) Pietrocola F, Izzo V, Niso-Santano M, Vacchelli E, Galluzzi L, et al. (2013) Regulation of autophagy by stress-responsive transcription factors. Semin Cancer Biol.

8) Otomo C, Metlagel Z, Takaesu G, Otomo T (2013) Structure of the human ATG12 ATG5 conjugate required for LC3 lipidation in autophagy. Nat Struct Mol Biol 20: 59-66.

9) Liu K, Czaja MJ (2013) Regulation of lipid stores and metabolism by lipophagy. Cell Death Differ 20: 3-11.

10) Isakson P, Holland P, Simonsen A (2013) The role of ALFY in selective autophagy. Cell Death Differ 20: 12-20.

11) Shaid S, Brandts CH, Serve H, Dikic I (2013) Ubiquitination and selective autophagy. Cell Death Differ 20: 21-30.

12) Ashrafi G, Schwarz TL (2013) The pathways of mitophagy for quality control and clearance of mitochondria. Cell Death Differ 20: 31-42.

13) Mizushima N, Yoshimori T, Levine B (2010) Methods in mammalian autophagy research. Cell 140: 313-326.

14) Klionsky DJ, Abeliovich H, Agostinis P, Agrawal DK, Aliev G, et al. (2008) Guidelines for the use and interpretation of assays for monitoring autophagy in higher eukaryotes. Autophagy 4: 151-175.

15) Zoladek A, Pascut FC, Patel P, Notingher I (2010) Non-invasive timecourse imaging of apoptotic cells by confocal Raman micro-spectroscopy. J Ram Spectrosc 42: 251-258.

16) Kunapareddy N, Freyer JP, Mourant JR (2008) Raman spectroscopic characterization of necrotic cell death. J Biomed Opt 13: 054002.

17) Verrier S, Notingher I, Polak JM, Hench LL (2004) In situ monitoring of cell death using Raman microspectroscopy. Biopolymers 74: 157-162.

18) Spowart JE, Townsend KN, Huwait H, Eshragh S, West NR, et al. (2012) The Autophagy Protein LC3A Correlates with Hypoxia and is a Prognostic Marker of Patient Survival in Clear Cell Ovarian Cancer. J Pathol 228: 437447.

19) Nijssen A, Bakker Schut TC, Heule F, Caspers PJ, Hayes DP, et al. (2002) Discriminating basal cell carcinoma from its surrounding tissue by Raman spectroscopy. J Invest Dermatol 119: 64-69.

20) de Jong BW, Schut TC, Maquelin K, van der Kwast T, Bangma CH, et al. (2006) Discrimination between nontumor bladder tissue and tumor by Raman spectroscopy. Anal Chem 78: 7761-7769

21) Brozek-Pluska B, Musial J, Kordek R, Bailo E, Dieing T, et al. (2012) Raman spectroscopy and imaging: applications in human breast cancer diagnosis. Analyst 137: 3773-3380.

22) Haka AS, Volynskaya Z, Gardecki JA, Nazemi J, Shenk R, et al. (2009) Diagnosing breast cancer using Raman spectroscopy: prospective analysis. J Biomed Opt 14: 054023.

23) Kamemoto LE, Misra AK, Sharma SK, Goodman MT, Luk H, et al. (2010) Near-infrared micro-Raman spectroscopy for in vitro detection of cervical cancer. Appl Spectrosc 64: 255-261. 
24) Short KW, Carpenter S, Freyer JP, Mourant JR (2005) Raman spectroscopy detects biochemical changes due to proliferation in mammalian cell cultures. Biophys J 88: 4274-4288.

25) Pascut FC, Kalra S, George V, Welch N, Denning C, et al. (2013) Noninvasive label-free monitoring the cardiac differentiation of human embryonic stem cells in-vitro by Raman spectroscopy. Biochim Biophys Acta 1830: 3517-3524.

26) Konorov SO, Jardon MA, Piret JM, Blades MW, Turner RF (2012) Raman microspectroscopy of live cells under autophagy-inducing conditions. Analyst 137: 4662-4668.

27) Shao J, Lin M, Li Y, Li X, Liu J, et al. (2012) In vivo blood glucose quantification using Raman spectroscopy. PLoS One 7: e48127.

28) Lui H, Zhao J, McLean D, Zeng H (2012) Real-time Raman spectroscopy for in vivo skin cancer diagnosis. Cancer Res 72: 2491-2500.

29) Bergholt MS, Zheng W, Lin K, Ho KY, Teh M, et al. (2011) Characterizing variability in in vivo Raman spectra of different anatomical locations in the upper gastrointestinal tract toward cancer detection. J Biomed Opt 16: 037003.
30) Schulze HG, Konorov SO, Piret JM, Blades MW, Turner RF (2013) Labelfree imaging of mammalian cell nucleoli by Raman microspectroscopy. Analyst 138: 3416-3423.

31) Barba I, Cabañas ME, Arus C (1999) The relationship between nuclear magnetic resonance-visible lipids, lipid droplets, and cell proliferation in cultured C6 cells. Cancer Res 59: 1861-1868.

32) Spector AA, Mathur SN, Kaduce TL, Hyman BT (1980) Lipid nutrition and metabolism of cultured mammalian cells. Prog Lipid Res 19: 155-186.

33) Bozza PT, Payne JL, Morham SG, Langenbach R, Smithies O, et al. (1996) Leukocyte lipid body formation and eicosanoid generation: cyclooxygenaseindependent inhibition by aspirin. Proc Natl Acad Sci USA 93: 11091-11096.

34) Di Vito M, Lenti L, Knijn A, Iorio E, D'Agostino F, et al. (2001) 1H NMRvisible mobile lipid domains correlate with cytoplasmic lipid bodies in apoptotic T-lymphoblastoid cells. Biochim Biophys Acta 1530: 47-66.

35) Pyrgiotakis G, Kundakcioglu OE, Finton K, Pardalos PM, Powers K, et al. (2009) Cell death discrimination with Raman spectroscopy and support vector machines. Ann Biomed Eng 37: 1464-1473. 